\title{
Nutrition, Artificial Sweeteners
}

National Cancer Institute

\section{Source}

National Cancer Institute. Nutrition, Artificial Sweeteners. NCI Thesaurus. Code C16012.

Study of artificial sweeteners as potential carcinogens and in relation to general health. 